\title{
Utility of Toe-brachial Index for Diagnosis of Peripheral Artery Disease
}

\author{
Seong Chul Park, Chang Yong Choi, Young In Ha, Hyung Eun Yang \\ Department of Plastic and Reconstructive Surgery, Soonchunhyang University Gumi Hospital, Soonchunhyang University College of Medicine, \\ Gumi, Korea
}

Background The ankle brachial pressure index (ABI) is a simple, useful method for diagnosing peripheral artery disease (PAD). Although the ABI is an objective diagnostic method, it has limited reliability in certain scenarios. The aim of the present study was to determine the accuracy and reliability of the toe brachial index (TBI) as a diagnostic tool for detecting stenosis in PAD, associated with normal or low $A B I$ values.

Methods $A B I$ and $T B I$ values were measured in 15 patients with diabetic gangrene who were suspected of having lower extremity arterial insufficiency. The ABI and TBI values were measured using a device that allowed the simultaneous measurement of systolic blood pressure in the upper and lower extremities. In addition, the ABI and TBI values were compared pre- and post-angiography.

Results Patients with an $\mathrm{ABI}$ of 0.9-1.3 showed almost no difference between the 2 measurements. The patients with TBI $>0.6$ had no arterial insufficiency. The patients with TBI $<0.6$ required vascular intervention with ballooning. After the angiography, the gangrenous wounds decreased in size more rapidly than they did prior to the intervention.

Conclusions Our findings suggest that TBI is the method of choice for evaluating lower limb perfusion disorders. This result requires further studies of TBI in a larger number of patients. Future long-term studies should therefore evaluate the utility of TBI as a means of screening for PAD and the present findings should be regarded as preliminary outcomes.

Keywords Toe brachial index / Ankle brachial index / Peripheral arterial disease
Correspondence: Chang Yong Choi Department of Plastic and Reconstructive Surgery, Soonchunhyang University Gumi Hospital, Soonchunhyang University College of Medicine, 179 1gongdan-ro, Gumi 730-706, Korea Tel: +82-54-468-9150

Fax: +82-54-463-7504

E-mail: ccysy@hanmail.net
This article was presented at the 69th congress of the Korean Society of Plastic and Reconstructive Surgeons on November 11, 2011 in Seoul, Korea.

No potential conflict of interest relevant to this article was reported.

\section{INTRODUCTION}

The ankle brachial index (ABI), which is the ratio of ankle to brachial systolic blood pressure, is a simple useful method for the diagnosis of peripheral arterial disease $(\mathrm{PAD})$ and is a highly specific method for assessment of vascular risk in otherwise asymptomatic patients [1]. Although ABI is an objective diagnostic method, this method is less reliable in cases where the arteries may be calcified and, therefore, resistant to compression. There- fore, the application of this index to diabetic patients is considered questionable, given the prevalence of medial arterial calcification that results in falsely elevated $\mathrm{ABI}$ values [2]. The presence of media sclerosis produces falsely elevated pressure values, as the arterial wall becomes stiffer and resists compression.

A low ABI indicates PAD. There are many cases of peripheral artery stenosis even when the $\mathrm{ABI}$ is normal or elevated [2]. In such cases, great toe artery pressure is commonly advocated for calculation of the toe brachial index (TBI), as the vessels of the 
toes are generally unaffected by media sclerosis [2]. The use of the ABI has been reported in many studies, but its calculation has been inconsistent among studies. The aim of the present study was to determine the accuracy and reliability of the TBI as an alternative diagnostic tool for the detection of stenosis in $\mathrm{PAD}$ when the patient may have a normal or higher $\mathrm{ABI}$ value.

\section{METHODS}

A total of 30 limbs, representing 15 patients (12 men and 3 women) were studied (Table 1). Patients with either diabetic gangrene or symptoms of intermittent claudication were included in the study. However, patients previously diagnosed with cardiovascular disease or cerebrovascular incidents and already undergoing treatment were excluded.

In patients with suspected lower extremity arterial insufficien$\mathrm{cy}$, the $\mathrm{ABI}$ and $\mathrm{TBI}$ were measured using a device (Non-invasive vascular screening device VP-2000, Omron, Kyoto, Japan) that allows simultaneous systolic blood pressure measurements from both the upper and lower extremities (Fig. 1). The measure- ments were carried out by trained vascular nurses under optimal conditions. In order to obtain the most accurate results, the patients fasted for at least 2 hours prior to having measurements taken and were not permitted to consume any form of caffeine

\section{Fig. 1. Case 12}

A 60-year-old female with diabetic gangrene. (A) Initial wound finding. Diabetic gangrene on left foot, lateral side. (B) Left foot wound 3 months later. The wound size was reduced.

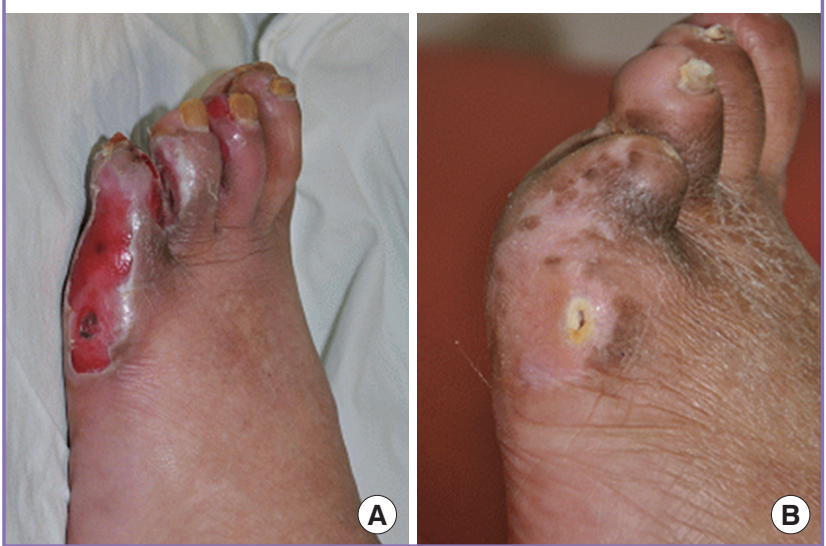

Table 1. Data of patients undergoing infrapopliteal angiography

\begin{tabular}{|c|c|c|c|c|c|c|}
\hline Cases & Lesion & $\mathrm{ABI}$ & TBI & Angiography & Ballooning & Wound management \\
\hline 1 & 1 right & 0.74 & 0.43 & ATA, PA & 0 & 2 joint amputation \\
\hline 2 & 1 left & 1.05 & 0.61 & Normal & & \\
\hline 3 & 2 right & 1.1 & 0.54 & ATA, PA & 0 & Skin graft \\
\hline 4 & 2 left & 1.1 & 0.65 & Normal & & \\
\hline 5 & 3 right & 0.99 & 0.31 & ATA, PA & 0 & 1 joint amputation \\
\hline 6 & 3 left & 1.01 & 0.63 & Normal & & \\
\hline 7 & 4 right & 0.84 & 0.62 & Normal & & \\
\hline 8 & 4 left & 0.91 & 0.45 & ATA, PTA & 0 & Skin graft \\
\hline 9 & 5 right & 0.89 & 0.54 & PA & 0 & 2 joint amputation \\
\hline 10 & 5 left & 0.96 & 0.92 & Normal & & \\
\hline 11 & 6 right & 1.03 & 0.56 & ATA & 0 & Wound revision \\
\hline 12 & 6 left & 1.13 & 0.57 & ATA & 0 & Local flap \\
\hline 13 & 7 right & 0.84 & 0.39 & ATA & 0 & Conservative treatment \\
\hline 14 & 7 left & 1.13 & 0.69 & Normal & & \\
\hline 15 & 8 right & 0.9 & 0.66 & Normal & & \\
\hline 16 & 8 left & 0.91 & 0.58 & PTA & 0 & 1 joint amputation \\
\hline 17 & 9 right & 0.86 & 0.31 & PTA & 0 & Local flap \\
\hline 18 & 9 left & 0.87 & 0.64 & Normal & & \\
\hline 19 & 10 right & 0.94 & 0.68 & Normal & & \\
\hline 20 & 10 left & 0.79 & 0.51 & PTA, PA & 0 & Local flap \\
\hline 21 & 11 right & 1.02 & 0.68 & Normal & & \\
\hline 22 & 11 left & 1 & 0.45 & ATA, PA & 0 & 2 joint amputation \\
\hline 23 & 12 right & 1.05 & 0.35 & PA, ATA & 0 & 1 joint amputation \\
\hline 24 & 12 left & 1.18 & 0.86 & Normal & & \\
\hline 25 & 13 right & 1.1 & 0.74 & Normal & & \\
\hline 26 & 13 left & 1.1 & 0.64 & Normal & & \\
\hline 27 & 14 right & 1.18 & 0.74 & Normal & & \\
\hline 28 & 14 left & 1.15 & 0.71 & Normal & & \\
\hline 29 & 15 right & 1.19 & 0.81 & Normal & & \\
\hline 30 & 15 left & 1.17 & 0.68 & Normal & & \\
\hline
\end{tabular}


for 3 hours prior to the evaluations. In addition, physical activity was limited during the 3-hour period before the evaluations and the patients were taken to the testing laboratory in a wheelchair. The ABI and TBI measurements were taken after the patients had lain still on a table for about 15 minutes. The laboratory temperature was also maintained at $22^{\circ} \mathrm{C}[1]$.

The ABI was measured by determining the systolic pressure in the brachial artery and in both the dorsalis pedis and posterior tibial arteries. The systolic pressures of each legs were divided by the brachial pressure [3]. Similarly, the TBI was calculated by dividing the systolic pressure of the great toe by that of the brachial artery [1]. Probes were attached to the tips of both great toes, and cuffs were placed on the arms and legs, above the ankle or at the base of the great toes. The higher of the two simultaneously measured brachial systolic blood pressure values was used in the analysis.

In patients diagnosed with arterial insufficiency, vessel dilation using a balloon catheter was performed. Patients with high TBI values were deemed to be candidates for this procedure. The balloon catheter was inserted through an incision in the inguinal area.

Patient wounds were treated by debriding the wound, minor amputations, or with skin grafts.

\section{RESULTS}

The patients in this study presented with either diabetic gangrene (14 patients) or intermittent claudication ( 1 patient). Patients with an $\mathrm{ABI}<0.9$ and/or a $\mathrm{TBI}<0.6$ in either leg were regarded as presenting with $\mathrm{PAD}$ and were considered for angiography. Some patients were clinically suspected of having PAD, but had ABIs within the normal range of 0.9 to 1.3 . These patients demonstrated almost no difference between the ABI values calculated using the systolic pressure from the dorsalis pedis and that calculated using the values from the posterior tibial artery. These patients also presented with diabetic gangrene or ulcers.

After calculating the $\mathrm{ABI}$ and $\mathrm{TBI}$, interventions were performed in 15 patients. Among the 30 legs evaluated, 17 were normal and 13 required intervention using an expandable balloon catheter. Low ABIs were detected in 2 legs of 12 patients. Normal ABIs and low TBIs were detected in 8 legs. A low ABI and low TBI were detected in 3 legs of 12 patients. Two legs from the 30 patients demonstrated an $\mathrm{ABI}<0.9$ but had a normal TBI, and the angiography was also normal.

No patient with a TBI $>0.6$ demonstrated arterial insufficiency, nor did they have findings consistent with medial sclerosis. The patients with the TBI $<0.6$ required vascular intervention with a balloon catheter. Four of these patients had lower extremity swelling, and 6 presented with pain after the vascular intervention. All 13 legs requiring vascular intervention had elevated temperatures in their extremities, and the patients were able to detect differences between the temperatures of their legs. Pinpoint bleeding was observed in the wound margin after debridement. Debridement was performed at least 2 to 3 times; 1 or 2 joint amputations were performed for 6 patients. The mean hospital stay was 55 days.

\section{Case reports}

Case 16

A 60-year-old female patient was referred to the vascular clinic with diabetic gangrene in her left foot. The patient had a left foot ABI of 0.91 and a TBI of 0.58 . Her gangrenous wound included dirty necrotic tissue and bone. The distal portion of her fifth toe had evidence of osteomyelitis. Debridement was repeated 3 times, and 1 joint amputation was performed. The mean values of both the ABI and TBI increased following angiography, with a concomitant reduction in the wound size (Fig. 1).

\section{Cases 5, 6}

A 69-year-old man was admitted with diabetic gangrene in his right foot. The patient had a measured right foot $\mathrm{ABI}$ of 0.99 , and a left foot $\mathrm{ABI}$ of 1.01; the right and left TBI values were 0.31 and 0.63 , respectively. This patient, with $\mathrm{PAD}$, was a candidate for angiography. An angiogram demonstrated complete occlusion of the distal peroneal artery and distal posterior tibial artery. Near total occlusion of the anterior tibial artery was also demonstrated by the angiogram. Intervention was performed using a balloon-expandable device. The post-angioplasty $\mathrm{ABI}$ and TBI were measured approximately 3 months later. The TBI value on

\section{Fig. 2. Cases 5, 6}

(A) Initial toe brachial index (TBI) values. The right and left TBI were 0.31 and 0.63 . (B) TBI values 3 months later. The values were elevated to 0.45 on the right and 0.66 on the left.

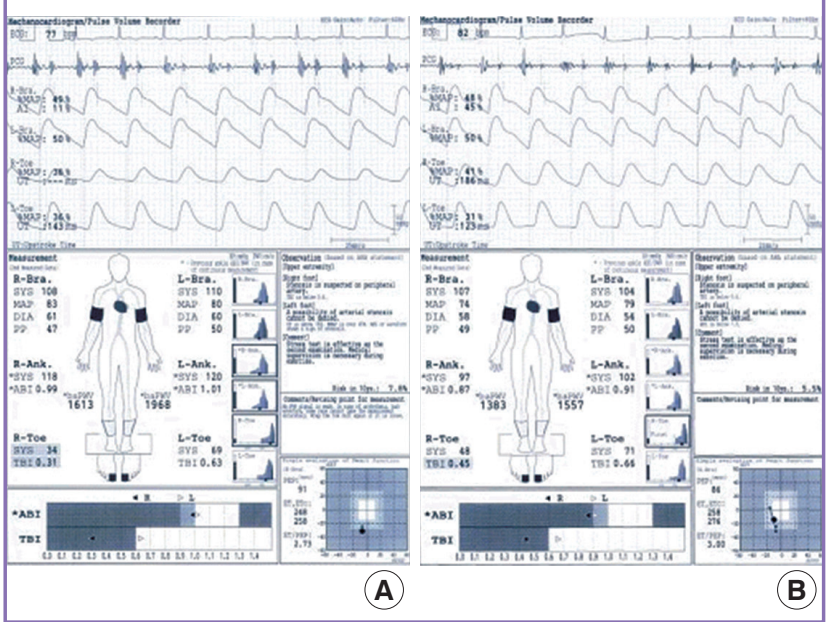




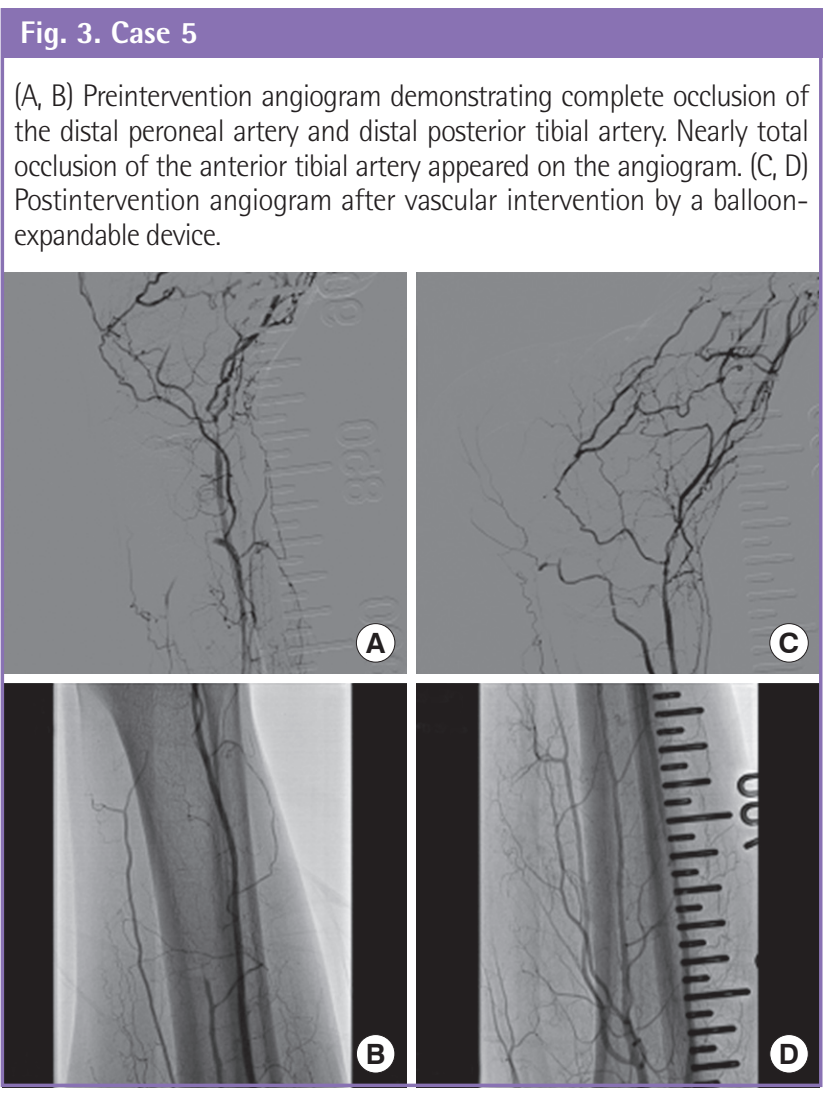

his right limb had increased to 0.45 from 0.41 (Figs. 2, 3).

\section{DISCUSSION}

PAD is a clinical manifestation of the atherosclerotic process. The prevalence of $\mathrm{PAD}$ has been evaluated in several epidemiological studies, and has been demonstrated to be as high as $20 \%$ in persons over 75 years of age [3]. PAD has been associated with increased cardiovascular morbidity and mortality, but only approximately one-third of PAD patients exhibit typical symptoms; the majority are asymptomatic [4]. It is vital, therefore, that general practitioners are able to accurately diagnose asymptomatic cases. Although vascular insufficiency can be diagnosed by either angiography or computed tomography angiograms, these invasive and expensive tests are not generally available to general practitioners. Therefore, a simple, non-invasive test, such as taking the $\mathrm{ABI}$ or $\mathrm{TBI}$, would be beneficial.

An $A B I$ value $<0.9$, an independent risk factor for cardiovascular disease, is caused by hemodynamically significant arterial stenosis and is most often used in epidemiological studies as a threshold value for the presence of PAD. However, in the presence of media sclerosis, the $\mathrm{ABI}$ is not a useful diagnostic tool. This is because the ABI increases due to arterial wall resistance to compression [2], tending to result in $\mathrm{ABI}$ values of 1.3 or
Fig. 4. Clinical course of diabetic gangrene

$D M$, diabetes mellitus; $A B I$, ankle brachial index; $T B I$, toe brachial index.

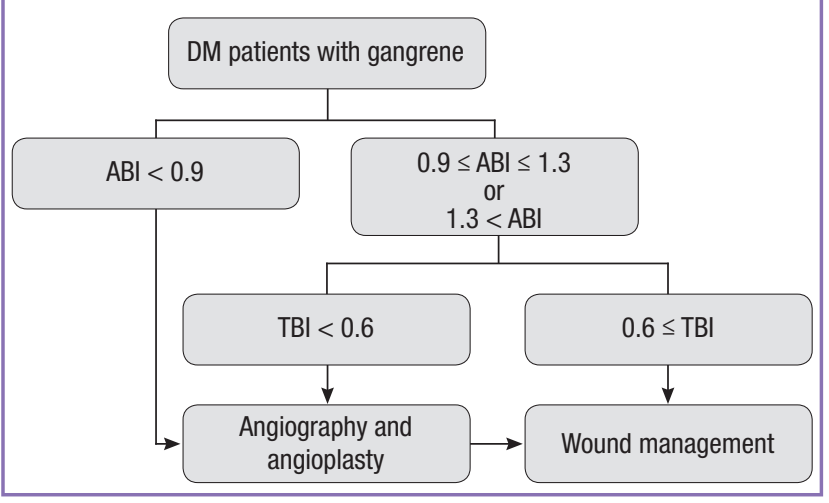

above. In addition, patients with chronic renal disease, a history of smoking, congestive heart disease, or other severe, lower extremity symptoms may have ABIs that are normal or above 1.3 [2]. In patients with PAD, media sclerosis does not typically affect the toe vessels. Therefore, TBI values are generally more accurate than $A B I$ in patients with media sclerosis.

In addition, many studies have indicated the utility of TBI values. Martin et al. evaluated the utility of TBI in diabetic patients and concluded that TBI was the method of choice for evaluating lower limb perfusion in the presence of overt arterial wall calcification [5]. Harrison et al. [1] also demonstrated that TBI is warranted in the assessment of PAD. The present findings also indicated that TBI was the preferred method for evaluating lower limb perfusion disorders, especially in patients with diabetic gangrene.

Thus, a treatment algorithm was devised for patients with diabetic gangrene. According to the algorithm, if the $A B I$ was less than 0.9 or if the TBI was lower than 0.6 after angiography, we performed wound management. In addition, in patients with normal $\mathrm{ABI}$ values, if the TBI was lower than 0.6 , angiography preceded wound treatment. Following this treatment algorithm, more rapid detection of $\mathrm{PAD}$ in patients with diabetic gangrene will be possible, allowing for improved wound management and reduced hospital stays (Fig. 4).

The American Diabetic Society recommends the evaluation of patients with diabetes mellitus for the presence of PAD at least every 5 years, even those patients with a normal ABI. As mentioned previously, media sclerosis in patients indicates the need for both $\mathrm{ABI}$ and $\mathrm{TBI}$ evaluations in order to detect PAD. Clinicians should also evaluate patients with diabetic gangrene using both the ABI and TBI, and we recommend our algorithm for the initial evaluation of these patients.

Verification of these results will require additional studies of the utility of TBI measurements in a larger number of patients. 
Future long-term studies should evaluate the utility of the TBI as a means of screening for PAD. In addition, larger clinical trials are also required to determine the utility of our recommended planning algorithm.

\section{REFERENCES}

1. Harrison ML, Lin HF, Blakely DW, et al. Preliminary assessment of an automatic screening device for peripheral arterial disease using ankle-brachial and toe-brachial indices. Blood Press Monit 2011;16:138-41.

2. Suominen V, Rantanen T, Venermo M, et al. Prevalence and risk factors of $\mathrm{PAD}$ among patients with elevated $\mathrm{ABI}$. Eur J Vasc Endovasc Surg 2008;35:709-14.

3. Diehm C, Schuster A, Allenberg JR, et al. High prevalence of peripheral arterial disease and co-morbidity in 6880 primary care patients: cross-sectional study. Atherosclerosis 2004;172:95-105.

4. Hirsch AT, Criqui MH, Treat-Jacobson D, et al. Peripheral arterial disease detection, awareness, and treatment in primary care. JAMA 2001;286:1317-24.

5. Martin Borge V, Herranz de la Morena L, Castro Dufourny I, et al. Peripheral arterial disease in diabetic patients: utility of the toe-brachial index. Med Clin (Barc) 2008;130:611-2. 